\title{
COMO O ESPECIALISTA EM ORTOPEDIA E TRAUMATOLOGIA AVALIA O ATENDIMENTO AO TRAUMA ORTOPÉDICO NO BRASIL
}

\author{
HOW DO ORTHOPEDIC SURGEONS RATE THE ORTHOPEDIC TRAUMA CARE IN BRAZIL
}

Jorge Santos Silva', Mauricio Kfuri Jr. ${ }^{2}$, Marcelo Abagge ${ }^{3}$, João Matheus Guimarães ${ }^{4}$, Paulo Roberto Lourenço Barbosa ${ }^{5}$, Daniel Balbachevsky ${ }^{6}$, Ralph Christian ${ }^{7}$, Kodi Kojima ${ }^{1}$

\section{RESUMO}

Objetivo: Apresentar os resultados de pesquisa Datafolha, realizada no período de 23 de setembro a 18 de outubro de 2010 , sobre as condições existentes para o exercício profissional na área do trauma ortopédico no Brasil. Métodos: pesquisa quantitativa, com abordagem telefônica dos entrevistados, por meio de sorteio aleatório de membros da Sociedade Brasileira de Ortopedia e Traumatologia, em cadastro contendo mais de 7.000 nomes. As entrevistas foram realizadas mediante aplicação de questionário estruturado, com aproximadamente 25 minutos de duração. Resultados: 97\% dos entrevistados dedica parte do seu tempo ao trauma ortopédico. $87 \%$ dos entrevistados exercem outra sub-especialidade, que não o trauma ortopédico. Na média dos atendimentos no país, $43 \%$ dos pacientes pertencem à rede pública de saúde e $41 \%$ pertencem à rede de convênios. O uso de implantes importados ocorre na minoria das situações (36\%) e $83 \%$ dos médicos que utilizam ambos os tipos de implantes julga que os nacionais apresentam qualidade inferior. $61 \%$ dos entrevistados julga a qualidade do atendimento em serviços públicos regular, ruim ou péssima. Metade dos entrevistados declara ter problemas para a liberação de suas solicitações de procedimentos junto aos planos de saúde em pelo menos $25 \%$ das vezes em que encaminham tais pedidos. Conclusão: $\mathrm{O}$ trauma ortopédico é uma especialidade exercida pela grande maioria dos ortopedistas brasileiros. A estrutura dos serviços públicos é considerada insatisfatória pela maioria dos ortopedistas entrevistados. A maioria dos ortopedista deseja uma reformulação nos honorários médicos e na infra-estrutura de serviços.

\section{ABSTRACT}

Objectives: The aim of this article is to present the data collected by Datafolha institute, from September 23rd. through October 18th. 2010 about orthopedic trauma care in Brazil. Method: A quantitative analysis based on telephonic interviews has been performed. From Brazilian Orthopedic Society database containing more than 7000 records. A structured query has been applied and the interview lasted around 25 minutes. Results: 97\% of interviewees dedicate part of his/her time to orthopedic trauma. $87 \%$ of all interviewees dedicate his/her time to more than one sub-specialty. The majority of orthopedic trauma patients comes from government insurance system (43\%), while $41 \%$ of patients come from private insurance. $61 \%$ of all interviewees think that the quality of public health system could be rated as unsatisfactory. Northeast of Brazil is the place where the majority of patients are from public health system and where we have highest rates of dissatisfaction (85\%) related to available infrastructure for orthopedic trauma care. Half of all interviewed individuals have problems for getting private insurance authorization previously to a surgery. Conclusions: Orthopedic trauma is a specialty practiced by the vast majority of orthopedic surgeons in our country. Neither the infrastructure nor the salaries satisfy the majority or orthopedic surgeons dedicated to trauma care.

Keywords: Trauma; Orthopedics; Infrastructure

1 - Instituto de Ortopedia e Traumatologia da Faculdade de Medicina - USP

2 - Serviço de Ortopedia da Faculdade de Medicina de Ribeirão Preto - USP

3 - Hospital do Trabalhador - Universidade Federal do Paraná

4 - Instituto Nacional de Traumatologia e Ortopedia - RJ

5 - Hospital Ipanema - Rio de Janeiro

6 - Departamento de Ortopedia da Escola Paulista de Medicina - UNIFESP

7 - Serviço de Ortopedia da Santa Casa de São Paulo

Trabalho realizado pela Sociedade Brasileira de Trauma Ortopédico

Correspondência: Alameda Lorena $n^{\circ} 427-14^{\circ}$ andar, Conjunto b - Jardim Paulista - CEP 01424-000.

Trabalho recebido para publicação: 15/03/11, aceito para publicação: 15/04/11. 


\section{INTRODUÇÃO}

As internações de pacientes por causas externas no Brasil, onde se inclui o traumatismo ortopédico, sofreram um aumento de $25,5 \%$ no período de 2008 a 2010 , passando de 769.001, em 2008, para 924.827 no ano de 2010, conforme dados do DATASUS. ${ }^{(1)}$ Este volume de hospitalizações está relacionado diretamente com o aumento do número de atendimentos de urgência e emergência, sobrecarregando um sistema já sabidamente deficiente.

Pronto-Socorros e Unidades atendimento de emergência lotadas de pacientes, assim como as dificuldades encontradas pelos médicos para lidar com tais situações, são reportadas praticamente todas as semanas pelas diferentes formas de mídia, em todas as regiões do nosso país.

Tentando avaliar como está a assistência aos pacientes com traumatismo ortopédico, a Diretoria da Sociedade Brasileira do Trauma Ortopédico (SBTO) estabeleceu como uma de suas prioridades, no ano de 2010, a realização de uma pesquisa nacional com os médicos ortopedistas, com o objetivo de conhecer a opinião desses especialistas sobre o atendimento ao traumatismo ortopédico tanto no Sistema Único de Saúde quanto na Saúde Suplementar.

\section{MÉTODO}

Realizou-se uma pesquisa quantitativa, com abordagem telefônica dos entrevistados, por meio de sorteio aleatório de membros da SBOT, em cadastro contendo aproximadamente 7.000 nomes. As entrevistas foram realizadas mediante aplicação de questionário estruturado, com aproximadamente 25 minutos de duração.

A coleta de dados ocorreu entre os dias 23 de setembro e 18 de outubro de 2010. A amostra final foi de 201 entrevistas com membros da SBOT, com a distribuição apresentada na Tabela 1. A proporção de entrevistados por região foi similar à observada em listagem fornecida pela SBOT, ou seja $60 \%$ dos entrevistados $(n=117)$ foram da região Sudeste, $16 \%(n=34)$ da região $\mathrm{Sul}$ e assim sucessivamente.

Foram utilizadas duas escalas de avaliação, dependendo dos quesitos considerados. A primeira de zero (menor nota) a 10 pontos (maior nota), considerando-se as notas cinco e seis como pontos médios. A segunda variando de um (menor nota) a cinco (maior nota), sendo a nota três considerada o ponto médio.
Tabela 1 - Número de membros da SBOT e distribuição dos entrevistados por região geográfica - Frequência absoluta e relativa.

\begin{tabular}{c|c|c|c|c}
\hline & $\begin{array}{c}\text { Membros da } \\
\text { SBOT }\end{array}$ & $\begin{array}{c}\text { Proporção } \\
(\%)\end{array}$ & Amostra & $\begin{array}{c}\text { Proporção } \\
(\%)\end{array}$ \\
\hline SUDESTE & 4.249 & 60 & 117 & 59 \\
\hline SUL & 1.161 & 16 & 34 & 16 \\
\hline NORDESTE & 918 & 13 & 25 & 12 \\
\hline $\begin{array}{c}\text { CENTRO- } \\
\text { OESTE }\end{array}$ & 539 & 7 & 15 & 7 \\
\hline NORTE & 248 & 3 & 9 & 4 \\
\hline TOTAL & 7.115 & 100 & $\mathbf{2 0 1}$ & 100 \\
\hline
\end{tabular}

As medidas estatísticas adotadas no estudo foram:

Média Aritmética Simples: É a medida de tendência central mais utilizada. O resultado é obtido através da soma de todos os elementos dividida pelo número de observações (amostra).

Moda: Representa o valor que aparece com maior freqüência em um conjunto de dados.

Mediana: A mediana é uma medida de localização do centro da distribuição dos dados, definida do seguinte modo: ordenados os elementos da amostra, a mediana é o valor (pertencente ou não à amostra) que a divide ao meio, isto é, $50 \%$ dos elementos da amostra são menores ou iguais à mediana e os outros $50 \%$ são maiores ou iguais à mediana.

A margem de erro máxima para o total da amostra foi de 7 pontos percentuais, para mais ou para menos, dentro de um nível de confiança de $95 \%$.

\section{RESULTADOS}

\section{Perfil demográfico da amostra}

Do total da amostra, 97\% atendem rotineiramente pacientes com traumatismo ortopédico. $\mathrm{O}$ gênero masculino é o predominante (97\%) e a idade média é de 42 anos.

\section{Grau de Satisfação}

$\mathrm{Na}$ avaliação de um (nada satisfeito) a 5 (muito satisfeito), cerca de $66 \%$ dos ortopedistas estão satisfeitos com a profissão (16\% muito satisfeitos, $49 \%$ satisfeitos, $22 \%$ mais ou menos satisfeitos, $10 \%$ pouco satisfeitos e $2 \%$ nada satisfeitos). A média das notas foi 3,7. Esta avaliação leva em conta não apenas o exercício 
dedicado ao trauma ortopédico mas à profissão medica com todas as suas facetas de exercício. $87 \%$ dos entrevistados exercem alguma outra sub-especialidade alem do trauma ortopédico.

Avaliando-se por regiões geográficas, os ortopedistas da região Sul (78\%) e da região Nordeste (76\%) são os mais satisfeitos com a profissão; e com relação ao tempo de exercício profissional, aqueles com tempo superior a 20 anos são os menos satisfeitos (58\% de satisfação) enquanto $68 \%$ daqueles com menos de 10 anos de atividade, consideram-se satisfeitos.

\section{Nível de especialização}

A média do tempo de conclusão da graduação foi de 17 anos. Todos os entrevistados possuem o título de especialista pela SBOT, $84 \%$ dos entrevistados têm a especialização em Ortopedia como a titulação máxima, $12 \%$ possuem Mestrado e 2\% Doutorado. Quanto às regiões geográficas, a região Sul é a que tem proporcionalmente o maior número de mestres $(18 \%)$ e de doutores (4\%).

Com relação ao exercício de outra especialidade na área da ortopedia, 20\% referem ser especialistas em Joelho, 12\% em Quadril, 12\% em Ombro e Cotovelo e $13 \%$ relatam somente o Trauma Ortopédico como a sua área exclusiva de atuação.

\section{Local e tempo dedicado ao atendimento ao traumatismo ortopédico}

Os ortopedistas (95\%) realizam atendimento de pacientes com traumatismo ortopédico em ambulatórios ou consultórios, $83 \%$ trabalham em pronto-socorros ou unidades de emergência e $92 \%$ realizam procedimentos cirúrgicos relacionados com a traumatologia. Ortopedistas que exercem atividade ambulatorial, em emergências e em centro cirúrgico correspondem a 75\% dos entrevistados.

O tempo dedicado em média à atividade ambulatorial ou de consultório é de 21 horas semanais (moda=20 horas/semana). Nos pronto-socorros os ortopedistas trabalham em média 26 horas por semana (moda $=24$ horas/ semana), e em média 15 horas por semana de atividade no centro cirúrgico (moda $=12$ horas/semana).

Os médicos formados há menos de 10 anos trabalham em média mais horas em pronto-socorros (31 horas/semana), enquanto os ortopedistas com mais de 20 anos de atividade dedicam mais tempo ao consultório (22 horas/semana).
$89 \%$ dos profissionais com menos de 10 anos de formados são os que mais sobrepõem os três tipos de atividade (consultório+pronto-socorro+centro cirúrgico), ao passo que esse fato só ocorre em $54 \%$ daqueles com mais de 20 anos de profissão.

\section{Número e tipo de atendimentos mensal}

Três situações clínicas de atendimento foram pesquisadas:

- pacientes com trauma ortopédico simples ou somente com uma fratura

- pacientes com politraumatismo agudo $(<2$ semanas do acidente)

- pacientes com politraumatismo tardio ( $>2$ semana do acidente)

Em um mês típico a média do número de pacientes com traumatismo ortopédico simples ou somente com uma fratura foi de 87 pacientes (moda=100). Pacientes com politraumatismo agudo $(<2$ semanas de acidente) foram em média $23($ moda $=20)$ e com politraumatismo considerado tardio, a média foi $14($ moda $=5) .79 \%$ dos ortopedistas referem que atendem pacientes com trauma ortopédico simples e também politraumatizados (agudo ou tardio).

Com relação à faixa etária observou-se que o menor número de atendimento nas três categorias pesquisadas ocorre entre os ortopedistas formados há mais de 20 anos.

\section{Rede de Atendimento}

Pesquisou-se onde ocorre o atendimento ao trauma ortopédico: rede pública, saúde suplementar ou particular. $75 \%$ dos ortopedistas o fazem na rede pública, $88 \%$ na saúde suplementar e $80 \%$ referem atendimento particular. 56\% dos ortopedistas atendem tanto na rede pública quanto na privada (suplementar + particular).

Com relação a origem dos pacientes: $43 \%$ dos atendimentos públicos, $41 \%$ dos atendimentos da saúde suplementar e a $16 \%$ dos particulares. É maior a proporção de atendimento a pacientes na rede pública (53\%). entre os profissionais com menos tempo de formatura $(<10$ anos).

\section{Tipos de implantes utilizados}

$80 \%$ utilizam implantes nacionais e importados. Apesar da maior proporção utilizada ser de implantes nacionais $(64 \%)$, a grande maioria $(83 \%)$ aponta inferioridade do material nacional em relação ao importado 


\section{Avaliação da Rede Pública de Atendimento}

$61 \%$ dos entrevistados avaliou de maneira negativa a infra-estrutura disponível para atendimento na rede pública. A principal queixa é relacionada à precariedade de equipamentos e implantes. Na região Nordeste a avaliação negativa foi ainda mais significativa, atingindo $85 \%$ dos entrevistados. O tempo médio de espera para operação de pacientes com fratura isolada do fêmur e antebraço é semelhante, em hospitais públicos e equivalente a 7 dias. Na região Nordeste este numero sobe, em media, para 10 dias.

\section{Avaliação da Rede Privada de Atendimento}

$68 \%$ dos entrevistados considera o atendimento ao trauma ortopédico nas redes privados como bom ou ótimo. Como pontos positivos na avaliação a disponibilidade de recursos técnicos e o acesso a uma maior variedade de implantes. Como ponto preocupante, a dificuldade em se autorizar os procedimentos cirúrgicos. Metade dos ortopedistas refere ter problemas neste sentido em pelo menos $25 \%$ de suas solicitações. O tempo médio de espera para o tratamento cirúrgico de uma fratura fechada do femur ou do antebraço nos hospitais privados no Brasil é de 3 dias.

\section{Percepção sobre o trauma ortopédico}

É consenso entre os profissionais entrevistados, que o trauma é um importante problema de saúde pública, que é relevante desenvolver programa de treinamento de médicos e que é necessário criar um sistema específico de atenção ao trauma. Duas necessidades foram apontadas pela maioria dos entrevistados. Em primeiro lugar, a necessidade de melhorar a remuneração do especialista dedicado ao atendimento do trauma ortopédico. Outra prioridade apontada é a necessidade de se investir mais recursos em centros de referencia em trauma ortopédico na rede pública do nosso pais. Organização de rede referência e contra-referência ao trauma é item mais mencionado como prioridade da SBTO, no Nordeste.
Cursos para educação continuada são mais solicitados no Norte e Centro-Oeste.

\section{Capacitação profisssional}

A grande maioria dos entrevistados refere frequentar os congressos da Sociedade Brasileira de Ortopedia e Traumatologia ou da Sociedade Brasileira de Trauma Ortopédico (91\%), como meio de se manter atualizado. $55 \%$ dos entrevistados não receberam treinamento para o atendimento geral ao trauma oferecido pelo Colégio Americano de Cirurgiões (ATLS ${ }^{\circledR}$ )

\section{CONCLUSÕES}

Os dados apresentados revelam que há disparidades entre as cinco principais regiões do pais quando se avalia infra-estrutura de atendimento e recursos disponíveis para o trauma ortopédico, especialmente na rede pública. Há uma tendência muito clara, nas cinco regiões, a julgar negativamente o atendimento ao trauma ortopédico na rede pública de saúde em todo o território nacional. Da mesma forma, entre os ortopedistas que tem acesso tanto a implantes nacionais quanto importados para o tratamento de fraturas, há uma avaliação negativa dos implantes produzidos em nosso pais. A atenção ao trauma ortopédico no ambiente de urgência é dada, em sua grande maioria, por profissionais mais jovens, com menor tempo de especialização e, portanto, com menor experiência. Há uma percepção clara de que investimentos precisam ser realizados para que o trauma ortopédico adquira o caráter de especialidade, com centros de referencia bem equipados na rede pública, melhor remuneração dos profissionais que se dedicam a esta área especifica da atenção medica e maior oferta de programas de educação e capacitação profissional.

\section{REFERÊNCIA}

1. http://www2.datasus.gov.br/DATASUS/index.php?area=0203 